\title{
Surface dose measurement with Gafchromic EBT3 film for intensity modulated radiotherapy technique
}

\author{
Ugur Akbas ${ }^{1, *}$, Nazmiye Donmez Kesen ${ }^{1}$, Canan Koksal $^{1}$, Murat Okutan $^{1}$, Bayram Demir ${ }^{2}$, Hatice Bilge Becerir ${ }^{1}$ \\ ${ }^{1}$ Istanbul University, Oncology Institute, Department of Medical Physics, Istanbul, Turkey \\ ${ }^{2}$ Istanbul University, Science Faculty, Department of Physics, Istanbul, Turkey
}

\begin{abstract}
Accurate dose measurement in the buildup region is extremely difficult. Studies have reported that treatment planning systems (TPS) cannot calculate surface dose accurately. The aim of the study was to compare the film measurements and TPS calculations for surface dose in head and neck cancer treatment using intensity modulated radiation therapy (IMRT). IMRT plans were generated for 5 head and neck cancer patients by using Varian Eclipse TPS. Quality assurance (QA) plans of these IMRT plans were created on rando phantoms for surface dose measurements. EBT3 films were cut in size of $2.5 \times 2.5$ $\mathrm{cm}^{2}$ and placed on the left side, right side and the center of larynx and then the films were irradiated with 6 MV photon beams. The measured doses were compared with TPS. The results of TPS calculations were found to be lower compared to the EBT3 film measurements at all selected points. The lack of surface dose calculation in TPS should be considered while evaluating the radiotherapy plans.
\end{abstract}

\section{Introduction}

The dose deposited between air and phantom is defined as the surface dose. The dose at the surface has two components; contaminant electrons from the air, the linac collimator, the scattering materials in the path of the beam and secondary electrons that arise from irradiated patient [1]. Surface dose measurement is a challenging issue, due to steep dose reduction in surface area because of the absence of electron equilibrium. This steep dose gradient is causing requirement of special measurement techniques with appropriate dosimetric devices.

The most accurate dosimetric device for surface dose measurement is extrapolation ionization chamber, yet it is not owned in all institutes. Instead of extrapolation chamber, parallel plate ionization chambers can be used after making Gerbi's overresponse corrections [2]. The overresponse occurs because of the scattering electrons from the sidewall of the parallel plate chamber. The physical properties of these chambers confine the usage with only phantom measurements.

Radiochromic film is suitable dosimetry for surface dose measurements with their high spatial resolution and low spectral sensitivity. Bilge et al. [3] used radiochromic EBT film and Markus parallel plate ionization chamber in their surface dose measurements; they found the difference between EBT film and ionization chamber to be within $5 \%$ for $6 \mathrm{MV}$ and $3 \%$ for $18 \mathrm{MV}$. Radiochromic films can be used as a good alternative to parallel plate chambers.
The treatment planning systems help us to receive the calculated doses at the related areas that irradiated with treatment plans. The prediction of surface dose with TPSs depends on some factors such as calculation algorithm, linac commissioning and beam modelling. Previous studies reported that TPSs still have not enough accuracy for surface dose calculations.

The aim of the study was to compare the film measurements and TPS calculations for surface dose in head and neck cancer treatment using intensity modulated radiation therapy.

\section{Materials and Methods}

Computed tomography (CT) images of 5 larynx cancer patients were acquired in head gantry-supine position. Thickness of the CT images was chosen $3 \mathrm{~mm}$. Gross tumor volume, clinical target volume, planning target volume and organs at risk were defined and contoured by radiation oncologist. Then images were sent to TPS. Treatment plans were created by using Eclipse 8.9 (Varian, Palo Alto, CA) TPS on Varian Trilogy linac equipped with a 120-leaf MLC, utilizing 6 MV coplanar photon beams. Dose calculation was performed with Analytical Anisotropic Algorithm (AAA). Calculation grid size was selected $2.5 \mathrm{~mm}$. IMRT plans were generated with 7 coplanar fields which were separated at $40^{\circ}$ apart. Sliding window technique was chosen for all plans and a fixed dose rate of $500 \mathrm{MU} / \mathrm{min}$ was applied for each field. QA plans were created using rando 
phantom for irradiation in same conditions. Gantry, collimator and couch positions set to same positions with treatment plans.

GafChromic EBT3 film (International Specialty Product, NJ, US) was utilized. The EBT3 film has a single active layer that sandwiched between two transparent polyester sheets. The EBT3 film is more sensitive than the older versions of radiochromic film with its wide dose range from 1 cGy to 40 Gy [4,5]. A calibration curve of film batch was created before irradiation (Fig. 1). The EBT3 film was prepared into size of $2.5 \times 2.5 \mathrm{~cm}^{2}$ and put perpendicularly between the RW3 water equivalent slab phantoms at the depth of $5 \mathrm{~cm}$ where the linac was calibrated with $1 \mathrm{cGy}$ equals to 1MU. The irradiation of EBT3 films were made with doses from $0-800 \mathrm{cGy}$ at a field size of $10 \times 10 \mathrm{~cm}^{2}$. One piece of EBT3 film was not irradiated to use as a background.
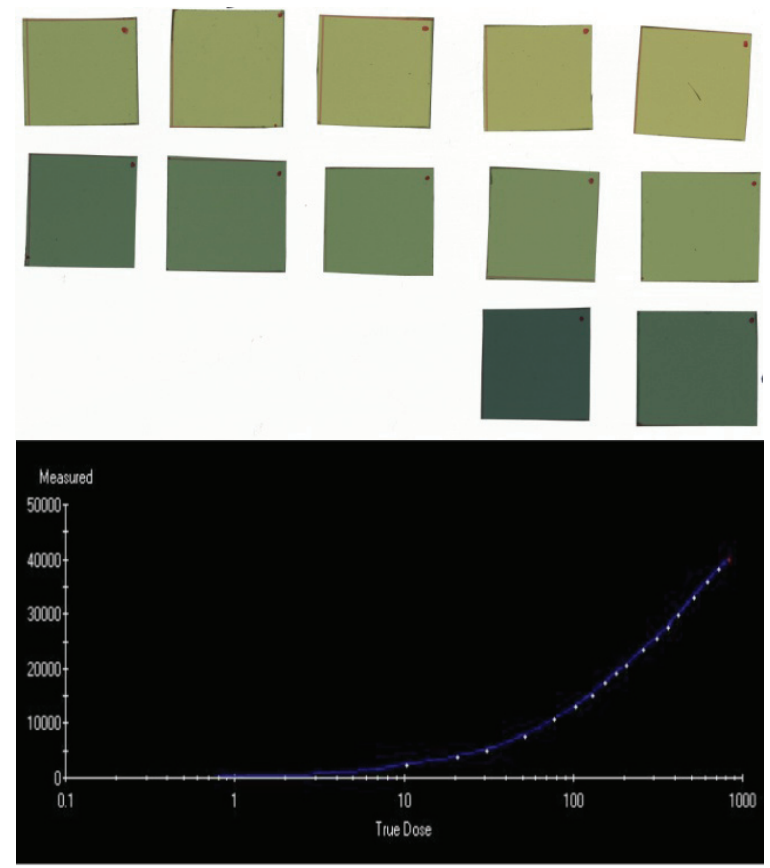

Fig. 1. The pieces of EBT3 film and the calibration curve of film batch

EBT3 films placed on the left side, right side and the center of larynx (Fig. 2). The films were irradiated with $6 \mathrm{MV}$ photon beams. The exposed films were scanned after 24 hours from irradiation and then the calibration curve of the film batch was used to obtain the absolute doses. The measured doses were compared with TPS.

In this study, the comparison of film measurements and TPS calculations were also performed in $10 \times 10$ $\mathrm{cm}^{2}$ open field using RW3 water equivalent slab phantoms for $6 \mathrm{MV}$ photon beams to evaluate surface and buildup region doses in different setup conditions (Fig. 3).

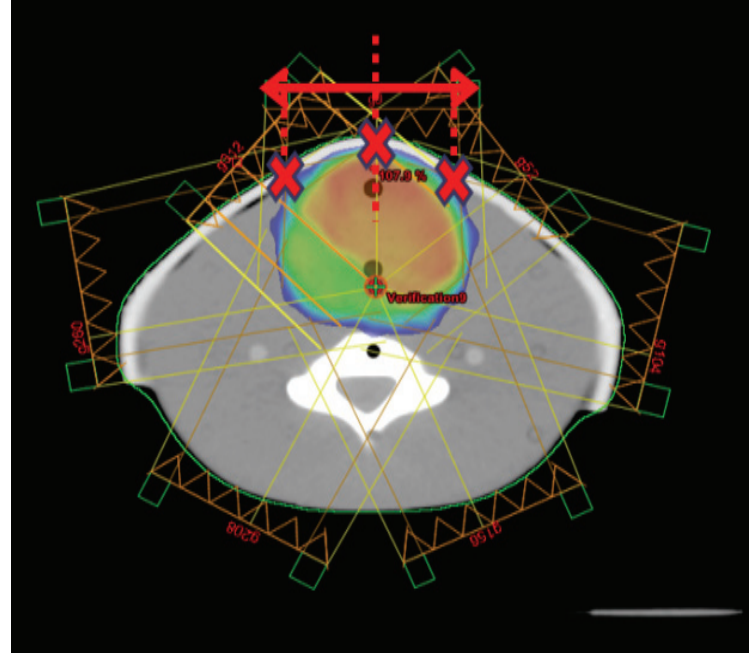

Fig. 2. Measurement points on rando phantom

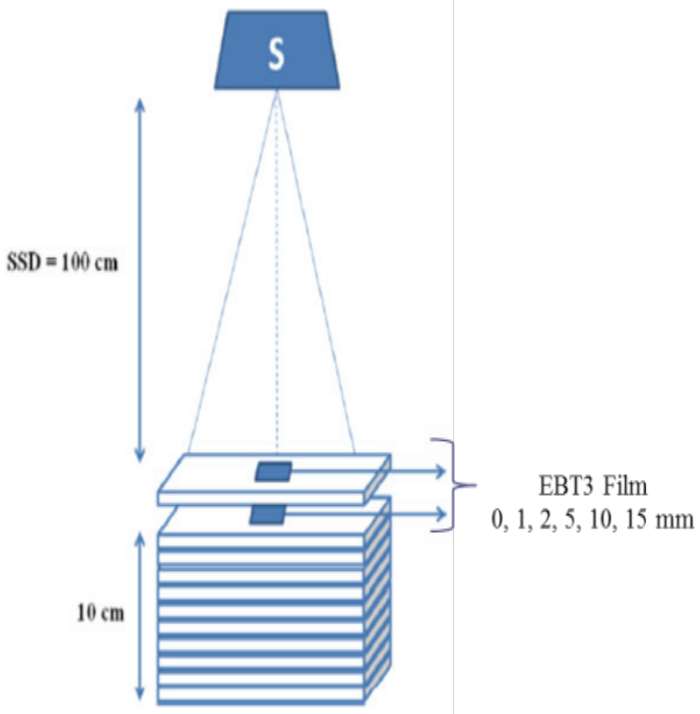

Fig. 3. Setup condition for open field irradiation

\section{Results}

The results of Gafchromic EBT3 film measurements and Varian Eclipse TPS calculations for 5 IMRT QA plans with 15 points (3 points for each) are shown in Fig.4. The surface doses were found to be higher with Gafchromic EBT3 film compared to Eclipse TPS for all points. The mean differences between measurements and calculations for $1^{\text {st }}, 2^{\text {nd }}, 3^{\text {rd }}, 4^{\text {th }}$ and $5^{\text {th }}$ IMRT plans were $29.3 \%, 25.4 \%, 20.8 \%, 29.9 \%$ and $23.6 \%$, respectively.

As mentioned before, in this study film measurements and TPS calculations compared in 10x10 $\mathrm{cm}^{2}$ open field. The difference of surface and buildup region doses of EBT3 film and TPS in open field is shown in Table 1. 


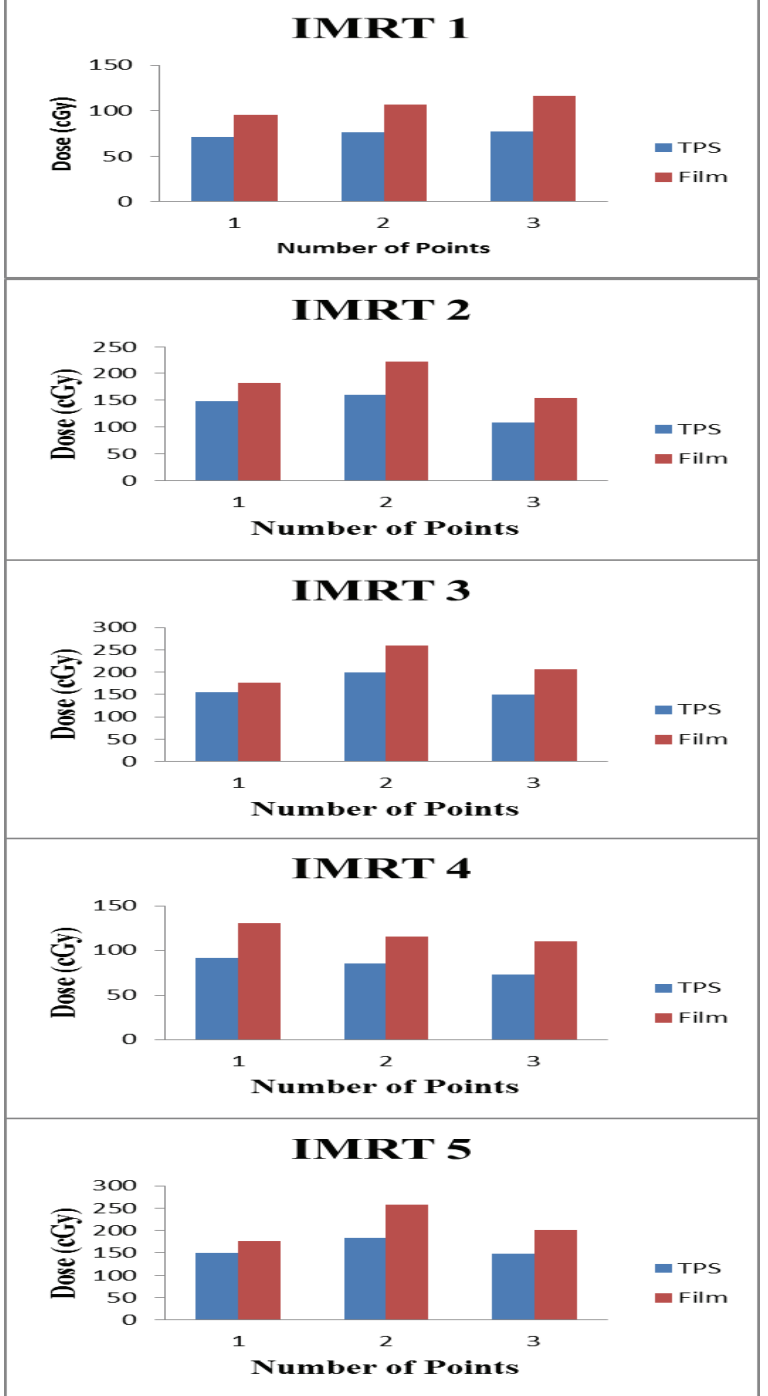

Fig. 4. The results of surface dose measurement and calculation for IMRT

Table 1. \%Depth dose (\%DD) values for open field

\begin{tabular}{|c|c|c|}
\hline $\begin{array}{c}\text { Depth } \\
\text { (cm) }\end{array}$ & $\begin{array}{c}\text { Gafchromic EBT3 } \\
\text { Film (\%DD) }\end{array}$ & $\begin{array}{c}\text { TPS } \\
\text { (\%DD) }\end{array}$ \\
\hline 0 & 20.4 & 11.0 \\
\hline 1 & 48.0 & 42.2 \\
\hline 2 & 61.3 & 54.5 \\
\hline 5 & 87.6 & 79.9 \\
\hline 10 & 99.2 & 95.8 \\
\hline 15 & 100.0 & 100.0 \\
\hline
\end{tabular}

\section{Conclusion}

Radiotherapy is an important treatment modality for the head and neck cancer. One of the most common intensity modulated technique, IMRT, has advantage of delivering maximum dose to target while sparing the healthy tissues. Immobilization, CT scanning, contouring the volumes of targets and normal tissues, technique of treatment planning, set-up conditions and dose delivery are the components of treatment. The dose deposited at surface in radiotherapy is very important in cases where the patient skin is defined as dose limiting tissue or part of the target in the irradiation area. An overexposure of the skin may give rise to acute reactions of the skin or the delayed effects of normal tissues.

The surface doses were found to be lower in TPS calculation compared to EBT3 film measurements both in IMRT and open field irradiations. The surface dose difference between TPS calculation and EBT3 measurement is greater in IMRT because of the using oblique fields. The studies reported that the surface dose increases with oblique angles; the relative dose might be $50 \%$ larger at an obliquity of $\sim 55^{\circ}[6,7]$.

Akino et al. [8] found that the measured dose with film dosimetry at $3 \mathrm{~mm}$ depth was higher than TPS calculation results by $15-30 \%$ for all treatment techniques but at deeper depths TPS calculation and film measurements results were found to be close. Devic et al. [4] reported that the even small thickness of EBT film can increase surface dose because of the steep dose gradient in the buildup region and should be accounted during calibration process. Chow et al. [9] compared the calculation accuracy of a superposition/convolution algorithm for oblique beams with Monte Carlo calculation, and reported that both the AAA and collapsed cone algorithms cannot accurately calculate dose distribution at depths less than $2 \mathrm{~mm}$.

Accurate knowledge of the dose at surface can be helpful to prevent the acute reactions and delayed effects. Our study has agreement with previous studies about the deficiency of TPS calculation in shallow depths. The lack of surface dose calculation in TPS should be considered while evaluating the radiotherapy plans.

\section{References}

1. A. Sigamani et al., Journal of Medical Physics/Association of Medical Physicists of India. 2016;41(2):85-91.

2. D. E. Mellenberg Jr. Medical Physics. 1990;17(6):1041-1044.

3. H. Bilge, A. Cakir, M. Okutan, H. Acar. Physica Medica. 2009;25(2):101-104.

4. S. Devic, et al., Medical Physics. 2006;33(4):1116-1124.

5. L.J. van Battum, D. Hoffmans, H. Piersma, S. Heukelom. Medical Physics, 2008;35(2):704-716.

6. F.M. Khan, The Physics of Radiation Therapy, Lippincott Williams \&Wilkins, Philadelphia, USA, 4th edition, 2010.

7. J.-P. Lin, T.-C. Chu, S.-Y. Lin, M.-T. Liu. Applied Radiation and Isotopes. 2001;55(3):383-391.

8. Y. Akino, I.J. Das, G. K. Bartlett, H. Zhang, E. Thompson, J.E. Zook. Medical Physics. 2013;40(1):011714 - 6 .

9. J. C. Chow, R. Jiang, M.K. Leung. Journal of Applied Clinical Medical Physics. 2010;12(1):3424. 